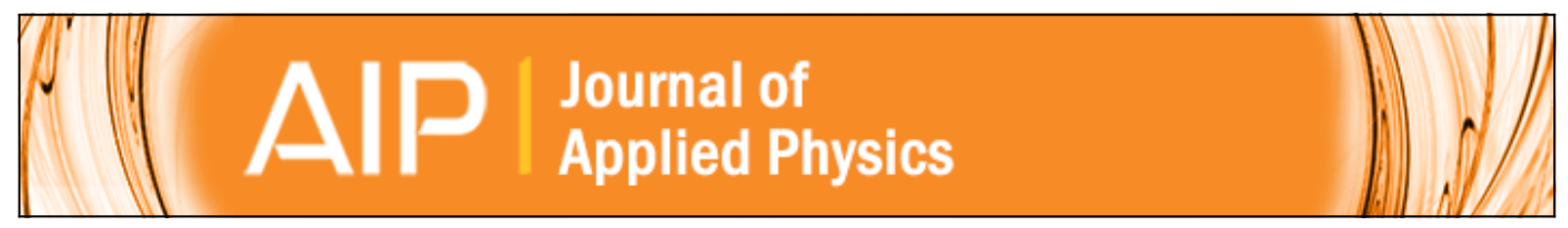

\title{
Modification of Al-oxide tunnel barriers with organic self-assembled monolayers
}

Salih Okur and John F. Zasadzinski

Citation: Journal of Applied Physics 85, 7256 (1999); doi: 10.1063/1.370541

View online: http://dx.doi.org/10.1063/1.370541

View Table of Contents: http://scitation.aip.org/content/aip/journal/jap/85/10?ver=pdfcov

Published by the AIP Publishing

\section{Articles you may be interested in}

Electron-beam-induced alteration of the dielectric properties of sandwiched self-assembled organic monolayers J. Appl. Phys. 107, 074103 (2010); 10.1063/1.3331988

The modification of self-assembled monolayer on indium tin oxide as cathode in inverted bottom-emitting organic light-emitting diodes

J. Appl. Phys. 104, 064506 (2008); 10.1063/1.2973466

Statistical representation of intrinsic electronic tunneling characteristics through alkyl self-assembled monolayers in nanowell device structures

J. Vac. Sci. Technol. B 26, 904 (2008); 10.1116/1.2905237

Phosphonate self-assembled monolayers on aluminum surfaces

J. Chem. Phys. 124, 174710 (2006); 10.1063/1.2186311

Transport studies of isolated molecular wires in self-assembled monolayer devices

J. Appl. Phys. 98, 034314 (2005); 10.1063/1.2005372

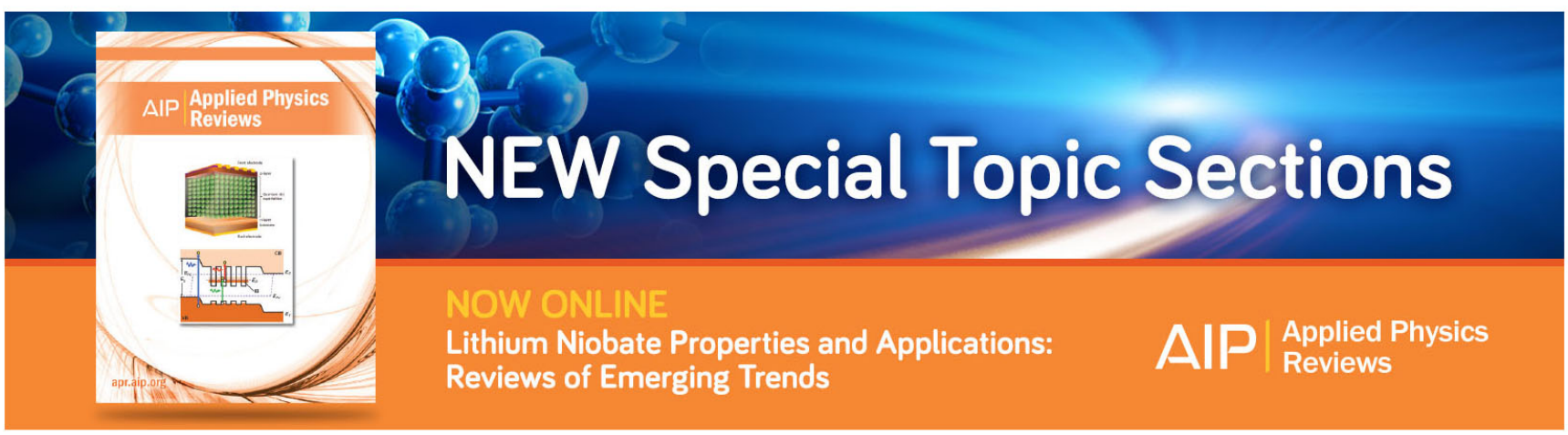




\title{
Modification of Al-oxide tunnel barriers with organic self-assembled monolayers
}

\author{
Salih Okur \\ Illinois Institute of Technology, Chicago, Illinois 60616 and Department of Physics, Izmir Institute of \\ Technology, TR-35230 Izmir, Turkey \\ John F. Zasadzinski ${ }^{\mathrm{a})}$ \\ Illinois Institute of Technology, Chicago, Illinois 60616
}

(Received 29 October 1998; accepted for publication 4 February 1999)

\begin{abstract}
Al-oxide tunneling barriers were modified by exposure to a vapor of $n$-octadecyltrimethoxysilane which forms self-assembled monolayers. The dynamic conductance $d I / d V$ of the modified Aloxide barrier between $\mathrm{Al}$ and $\mathrm{Pb}$ electrodes was measured at $4.2 \mathrm{~K}$. Quasilinear conductance backgrounds are observed up to $200 \mathrm{mV}$ with a strength that increases with increasing exposure time from 10 to $60 \mathrm{~min}$. A saturation effect is observed around $200 \mathrm{mV}$. Beyond $200 \mathrm{mV}$ the dynamic conductance shows a parabolic behavior indicative of elastic tunneling from an asymmetric barrier. The linear background is attributed to inelastic tunneling from a continuum of excitations. (C) 1999 American Institute of Physics. [S0021-8979(99)03910-9]
\end{abstract}

\section{INTRODUCTION}

Self-assembled monolayers (SAMs) are ordered molecular assemblies formed by the adsorption of an active surfactant on a solid surface. Two-dimensional order is a consequence of intermolecular forces but depends also on the length of the molecule and properties of the underlying surface. SAMs have a number of properties that are important for fundamental studies as well as applications, such as chemical sensors, ${ }^{1}$ novel photoresists, ${ }^{2}$ materials with enhanced nonlinear optical properties, interfacial electron transfer, ${ }^{1}$ molecular conductivity ${ }^{3}$ and surface wetting. ${ }^{4}$ There has been recent interest in these layers to protect the surfaces of high temperature superconductors (HTS'). ${ }^{5}$ Furthermore, the typical molecular lengths $(\sim 25 \AA)$ offer the possibility of utilizing a SAMs layer as an insulating barrier for tunnel junctions on HTS. The first such junctions were formed on thin films of $\mathrm{YBa}_{2} \mathrm{Cu}_{3} \mathrm{O}_{7}$ (YBCO) using octadecylamine as the organic molecule and a $\mathrm{Pb}$ counterelectrode. ${ }^{6,7}$ The results of this study were difficult to interpret due to the presence of a native barrier on the air-exposed surfaces of the YBCO film. Thus the observation of a strong, linearly increasing background in the dynamic conductance of these junctions and poorly defined superconducting gaps in YBCO could not be definitively attributed to the SAMs layer, as such effects were often observed in junctions without a SAMs layer.

The intention of this investigation was to examine the utility of SAMs as tunnel barriers on conventional superconductors. The well established elastic tunneling characteristics of superconducting $\mathrm{Pb}$ films make this material a natural choice for this study. Our initial attempts were to make superconductor-insulator-normal metal (SIN) sandwichtype junctions of the form $\mathrm{Pb} / \mathrm{SAMs} / \mathrm{Au}(\mathrm{Ag})$ by growing the SAMs layer directly on a deposited film of $\mathrm{Au}$ or $\mathrm{Ag}$, and

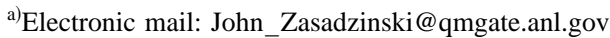

following the process with a thin film $\mathrm{Pb}$ counterelectrode. The organic used was octadecyltrimethoxysilane (OTMS) and the $\mathrm{Au}(\mathrm{Ag})$ film was dipped directly into the liquid. These junctions all resulted in electrical shorts, presumably due to pinholes in the SAMs layer. We thus used $\mathrm{Al}$ films as the base electrode which were then subsequently oxidized by a very short exposure $(\sim 10 \mathrm{~s})$ to pure oxygen gas. This provided a thin, surface layer of $\mathrm{Al}$ oxide upon which the SAMs were grown as shown in Fig. 1. A systematic study of junction properties was obtained by fitting the dynamic conductance $(d I / d V$ vs $V)$ to the elastic tunneling model of Brinkman, Dynes and Rowell. ${ }^{8}$ The principal result of this work is that there is a uniform increase in barrier thickness with exposure to the OTMS vapor, indicating that the organic layer is participating as a tunnel barrier, but there is also a relative increase in the contribution of another conductance channel. This additional channel is characterized by a strong, linear increase in conductance with bias voltage $(\sim|V|)$ up to a characteristic voltage $(\sim 170 \mathrm{mV})$ where saturation then sets in. As the relative contribution of this other channel increases, the superconducting gap structure of the $\mathrm{Pb}$ becomes increasingly broadened. While we have not unambiguously identified the origin of this second channel, it appears to be consistent with inelastic tunneling from a continuum of states.

\section{EXPERIMENTAL PROCEDURE}

A $99.99 \%$ purity aluminum pellet from Alfa Products was used and the $1 / 2 \mathrm{~cm}$ diam pellet was cut into small pieces. The Al pieces were cleaned using $0.5 \mathrm{M} \mathrm{NaOH}$ solution and etching for $1 \mathrm{~min}$. Then two or three cleaned $\mathrm{Al}$ pieces were put into a boron nitride crucible wrapped by tungsten filament. A clean glass microscope slide was placed roughly 8 in. from the source. An aluminum mask was placed close to the slide to make sharp edged $\mathrm{Al}$ strips. 1000 $\AA$ thick Al films were deposited on the microscope glass 


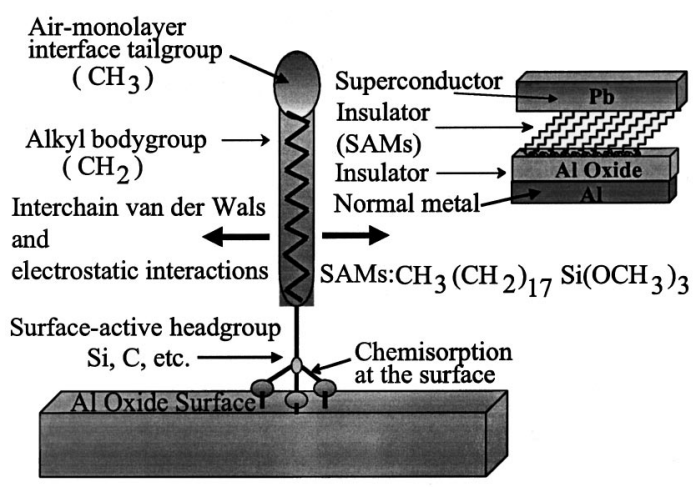

FIG. 1. A schematic view of a SAMs structure. Inset: A schematic view of an $\mathrm{Al} / \mathrm{Al}_{2} \mathrm{O}_{3} / \mathrm{SAMs} / \mathrm{Pb}$ tunnel junction.

slide with a deposition rate of $15 \AA / s$. The $\mathrm{Al}$ film was oxidized by venting the chamber to pure oxygen for $10 \mathrm{~s}$. This gave reproducible results with junction resistances between 1 and $40 \Omega$.

The next step was modifying the oxidized Al strips with the organic molecule, OTMS. The intention was to obtain a complete monolayer of chemisorbed molecules on the oxidized aluminum film. There are two basic methods for doing this: gas phase and liquid phase doping. In gas phase doping the oxidized aluminum strip is exposed to a vapor of the dopant in the vacuum chamber. ${ }^{9,10}$ If the substance is volatile, the vapor can be introduced from a bulb that is isolated from the vacuum chamber by a stopcock, or a sealed capillary glass can be broken inside the chamber.

In the case of liquid doping, ${ }^{11,12}$ the dopant molecules are applied to the oxidized Al film from a liquid solution. The concentration of molecules on the surface is controlled by varying the concentration of the dopant molecules in the solution. The entire slide can be put into a solution containing the molecules and the excess solution shaken or blown off. Dropping of the dopant solution on the junction area and spinning it off is another way of liquid doping. At first, we used the liquid phase doping method to make ( $\mathrm{Au}, \mathrm{Ag})-$ $\mathrm{SAMs}-\mathrm{Pb}$ tunnel junctions. We simply put freshly evaporated $\mathrm{Au}$ or $\mathrm{Ag}$ films into OTMS for 2 days to obtain maximum (complete) coverage and spin off the excess molecules. Our attempts to make pure SAMs barriers using the liquid doping procedure failed. Shorts through pinholes in the pure SAMs barrier did not allow a tunneling current to be measured.

In the liquid phase doping procedure the $\mathrm{Al}$ film must be taken out from the vacuum chamber and therefore exposed to air. This results in a thick Al-oxide layer that is affected by humidity and therefore uncontrolled. Because of these difficulties we chose gas phase doping to introduce the OTMS molecule and modified our vacuum system. ${ }^{13}$ We attached a magnetovacuum valve between the vacuum chamber and a vacuum hose with an open end. The open end was closed with a laboratory glass tube after filling with OTMS organic molecule. A pure oxygen tank was attached to the venting line of the chamber. The air inside the venting line was pumped with a mechanical pump and then filled with pure oxygen before each deposition. The air inside the hose at- tached to the OTMS molecule source was also pumped to the vacuum pressure of $10^{-6}$ Torr while the valve between chamber and OTMS tube was open. It was kept closed during $\mathrm{Al}$ deposition and oxidation. After $10 \mathrm{~s}$ oxidation the vacuum system was brought to $10^{-7}$ Torr.

By opening the valve between the vacuum chamber and the organic molecule, SAMs have been introduced as an additional barrier onto the $\mathrm{Al}$ oxide by exposure to the molecular vapor for $10-60 \mathrm{~min}$. After opening the valve to the organic molecule the chamber pressure suddenly rises from $10^{-7}$ Torr to $10 \mathrm{mTorr}$, and then it increases slowly with time. A heat gun was used to increase the vapor pressure of the OTMS since it starts to boil at about $400{ }^{\circ} \mathrm{C}$ at $50 \mathrm{mTorr}$. A copper tube was set up below the substrate and connected to the OTMS bulb to carry the vapor directly under the oxidized $\mathrm{Al}$ strips. The pressure difference between the organic liquid and the chamber helps to move the vapor of OTMS through the copper tube onto the $\mathrm{Al}$ oxide strips.

The chamber was repumped to $10^{-7}$ Torr for the $\mathrm{Pb}$ electrode (cross strip) deposition after finishing organic molecule doping. The substrate deposited with $\mathrm{Al}+\mathrm{Al}$ oxide $+\mathrm{SAMs}$ was moved over the cross strip mask. $\mathrm{Pb}$ evaporation was done without using a shutter so that $\mathrm{Pb}$ deposition rates started from zero. This minimizes knocking off the freshly doped organic molecules from the $\mathrm{Al}$-oxide film surface. A $2500 \AA \mathrm{Pb}$ deposition takes about $1 \mathrm{~min}$.

Thirty junctions are produced on a single microscope glass slide in our system, and only six junctions can be measured at a time. A set of six junctions is cut from the $1 \times 3$ in. glass microscope slide using a hard jaw spinning at 20000 rpm as a guide in making the scratch; then the slide is bent until it breaks along the scratch. After the junctions are removed from the vacuum evaporator, their resistance was measured immediately. The typical resistance for tunnel junctions was about $10 \Omega$ for a clean junction with a 0.5 $\times 0.5 \mathrm{~mm}^{2}$ area but increased up to $5000 \Omega$ with exposure to OTMS vapor. Before mounting the junctions to the sample holder, the ends of the strips are soldered with indium to make connections to thin insulated copper wires. The sampler holder was designed to fit down the $5 / 8$ in. neck of a commercial liquid helium storage Dewar. In general it is important to get the junctions into liquid helium within 20 min after removing them from the vacuum system to prevent degradation.

Four terminal measurement was used to minimize the effect of the strip resistance on the measured junction characteristics. After the junctions are inserted into liquid helium (at $4.2 \mathrm{~K}$ ), it is important to measure the current-voltage $(I-V)$ characteristics out to a few $\mathrm{mV}$ of bias to determine if the junctions are good or if there are: (i) bad electrical connections, (ii) excessive noise, and (iii) poor-quality junctions. This is done by looking at the structure due to the superconducting $\mathrm{Pb}$ energy gap. For an ideal conductance at $4.2 \mathrm{~K}$, it is important to note that the conductance at zero voltage should be approximately $1 / 7$ the conductance at high bias voltage. The conductance at zero bias is due to thermal excitations above the $\mathrm{Pb}$ gap at $4.2 \mathrm{~K}$. If the ratio is closer to unity, it indicates current flow in channels other than elastic tunneling such as microshorts. In this experiment, a lock-in 


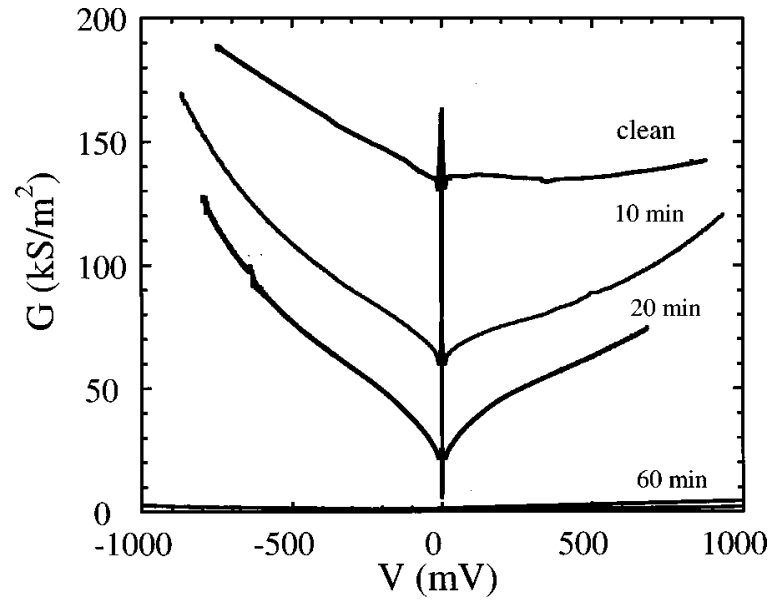

FIG. 2. The representative tunneling conductance vs bias voltage curves for clean and modified $\mathrm{Al}$-oxide barriers exposed to SAMs for 10-60 min.

amplifier was used to measure first and second derivatives using standard harmonic detection methods.

\section{RESULTS AND ANALYSIS}

The tunneling conductance spectra $(d I / d V$ vs $V)$ were obtained for clean junctions (no SAMs layer) and for junctions formed with three different exposure times to the OTMS vapor, 10, 20 and 60 min. Representative conductance spectra at $4.2 \mathrm{~K}$ for the four different junction types are shown in Fig. 2 out to $1 \mathrm{~V}$ bias and on this voltage scale the superconducting energy gap feature of $\mathrm{Pb}$ is barely discernible. For each junction type, up to 20 junctions were measured. The characteristic features of the clean junction are typical of those found in the literature for $\mathrm{Al} / \mathrm{Al}_{2} \mathrm{O}_{3} / \mathrm{Pb}$ thin film junctions. Near zero bias, but above the $\mathrm{Pb}$ gap voltage, the conductance is flat and at high bias the conductance has an asymmetric, parabolic shape. These features are consistent with elastic tunneling through a trapezoidal barrier. With increasing exposure time to the OTMS vapor, the junction conductance at zero bias decreases substantially, indicating that the tunnel barrier thickness is increasing. Furthermore, there is a noticeable change in the shape of the high bias, background conductance. For bias voltages $|V|<300 \mathrm{mV}$, there is a rapidly increasing, concave downward conductance which is not consistent with elastic tunneling and suggests that an additional conductance channel is developing.

These features can be seen more clearly in Fig. 3 where rescaled junction conductances are presented. For each curve, the conductance $G(V)$ has been divided by the conductance value at $2 \mathrm{mV}$ bias, $G(2 \mathrm{mV})$. On this rescaled plot the 60 min exposure junction which was barely observable in Fig. 1 exhibits the most rapid relative increase in conductance with bias voltage. The junctions all display a strong, quasilinear increase in conductance out to about $\pm 100 \mathrm{mV}$, followed by a concave downward shape out to $\pm 300 \mathrm{mV}$, followed by an increasing, parabolic shape for $|V|$ beyond $300 \mathrm{mV}$. Since elastic tunneling predicts a flat conductance for voltages much less than the barrier height, we attribute the strong, linear increasing conductance to an additional conductance channel. The background tunneling conduc-

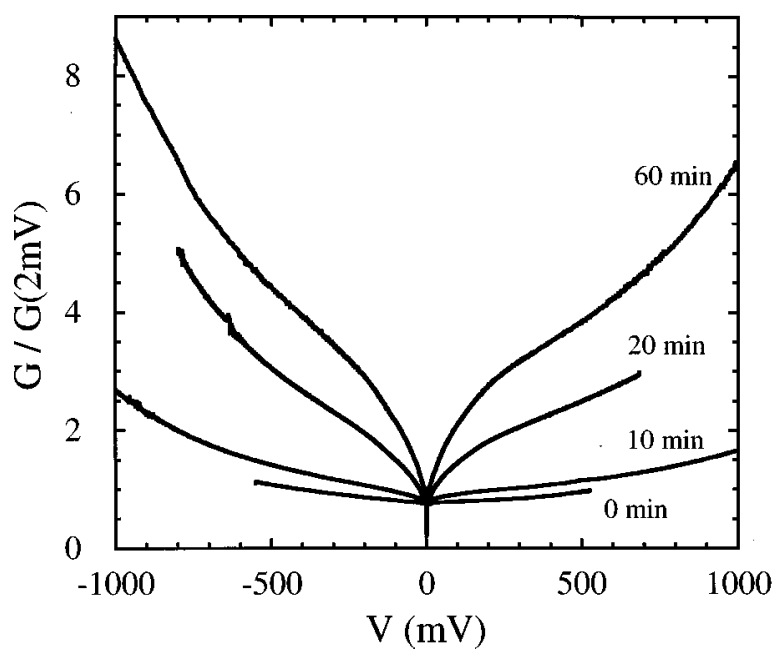

FIG. 3. Normalized dynamic tunneling conductance vs bias voltage (high bias) for modified Al-oxide barriers exposed to SAMs for 60, 2010 and 0 $\min$ (from top).

tances of high temperature superconductors often exhibit a similar linearly increasing background shape that has been attributed to inelastic tunneling from a continuum of states. Kirtley $^{14,15}$ has shown that such linear increases in background can be consistently observed in modified Al-oxide barriers when magnetic impurities have been introduced, but it is not clear whether such magnetic ions develop when the OTMS molecule attaches itself to the Al-oxide surface. Nevertheless, we will analyze these data within the framework of continuum inelastic tunneling, because this channel offers a natural explanation of the concave downward shape as being due to a saturation effect. When the continuum of states has a cutoff, as in a phonon spectrum, the inelastic channel approaches a constant at high bias. ${ }^{13}$ This will be discussed in more detail later in the article.

In Fig. 4 is shown the rescaled conductance data for bias voltages near the superconducting energy gap of $\mathrm{Pb}$. The clean junction exhibits a well-defined superconducting gap feature with sharp conductance peaks near the $\mathrm{Pb}$ energy

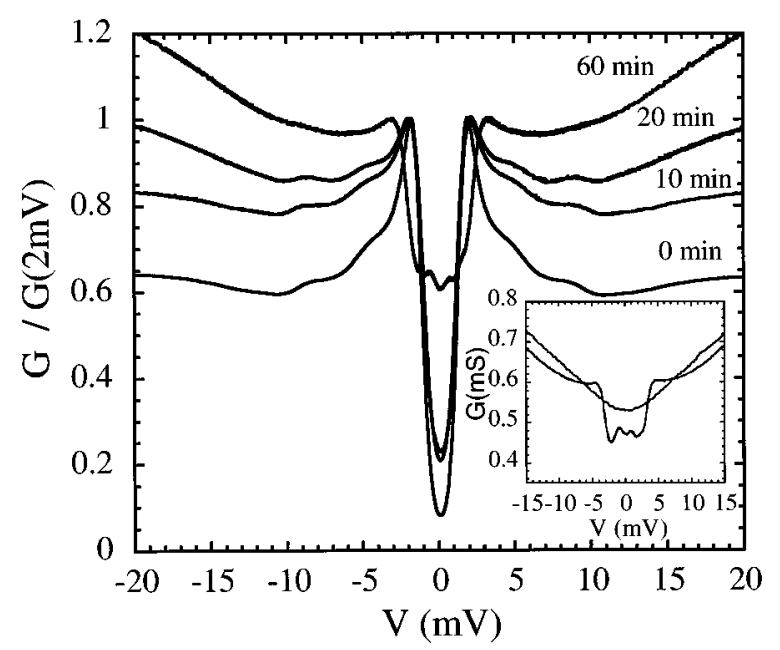

FIG. 4. The normalized conductance at low bias voltages (less than $20 \mathrm{mV}$ ). Inset: One of the $d I / d V$ vs $V$ curves for the 60 min exposed tunnel junctions at 4.2 and at $8 \mathrm{~K}$. 
gap, $\Delta=1.4 \mathrm{meV}$. Also, the strong coupling effects due to peaks in $\mathrm{Pb}$ phonon density of states are seen near 5 and 10 $\mathrm{meV}$. With increasing exposure to the OTMS vapor, the $\mathrm{Pb}$ gap structure weakens and the strong linear background becomes observable. This is further evidence that the SAMs layer is not just modifying the barrier properties (e.g., barrier height and thickness) as this would lead to nearly identical rescaled conductance spectra in the $\mathrm{Pb}$ gap region. Rather, in correlation with the development of the linear background there is a weakening and broadening of the $\mathrm{Pb}$ gap structure, which indicates that another conductance channel is contributing to the current. For the maximum exposure $(60 \mathrm{~min})$ there is often observed an apparent increase of the $\mathrm{Pb}$ gap feature as seen in Fig. 4 and an additional, smaller, gap-like feature. Both structures disappear above the $T_{c}$ of $\mathrm{Pb}$ as shown in the inset of Fig. 4 and thus this indicates that a multiple SISIN junction has formed. Presumably, some $\mathrm{Pb}$ atoms have diffused down the OTMS molecule and a junction is formed between a cluster of $\mathrm{Pb}$ atoms and the top $\mathrm{Pb}$ film with the SAMs layer acting as the tunnel barrier. The two features are the sum and difference gaps of the $\mathrm{Pb}$ clusters and $\mathrm{Pb}$ layer.

The parabolic increase in tunneling conductance background above $200 \mathrm{mV}$ can be explained as elastic tunneling through asymmetrical barriers given by Brinkman, Dynes and Rowell (BDR) in $1970 .{ }^{8}$ According to this theory the tunneling conductance through a trapezoidal barrier is given as

$$
\frac{G_{\mathrm{el}}(V)}{G(0)}=1-b V+c V^{2} \quad \text { (elastic tunneling), }
$$

where $G(0)$ is the zero bias conductance and $b$ $=A_{0} \Delta \phi /\left(16 \bar{\phi}^{3 / 2}\right)$ and $c=9 A_{0}^{2} /(28 \bar{\phi})$ are the elastic tunneling fitting parameters described below. To account for the other conductance channel which we have labeled "inelastic tunneling", we add a term

$$
\frac{G_{\text {inel }}(V)}{G(0)}=\alpha \tanh \left(|V| / V_{c}\right) .
$$

This phenomenological expression exhibits the essential features observed in Fig. 3, i.e., a linear increase with $V$ for $V$ $\ll V_{c}$, and constant (or saturation) conductance for $V \gg V_{c}$. It has been demonstrated both experimentally ${ }^{14}$ and theoretically ${ }^{15}$ that a linear increase in conductance with bias results from inelastic tunneling of electrons via a continuum of states, say from phonons or spin fluctuations. However, when the spectrum of excitations is exhausted at very high bias, the conductance will saturate to a constant value. Thus, our phenomenological expression is physically quite reasonable.

The data fitting function describing the elastic and inelastic tunneling process is given as

$$
\frac{G(V)}{G(0)}=1-b V+c V^{2}+\alpha \tanh \left(|V| / V_{c}\right) .
$$

The best values for the fitting parameters $b, c$ and $\alpha$ for each set of tunneling data have been calculated by using the least square fit method, and the cutoff energy $E_{c}=e V_{c}$ for inelas-

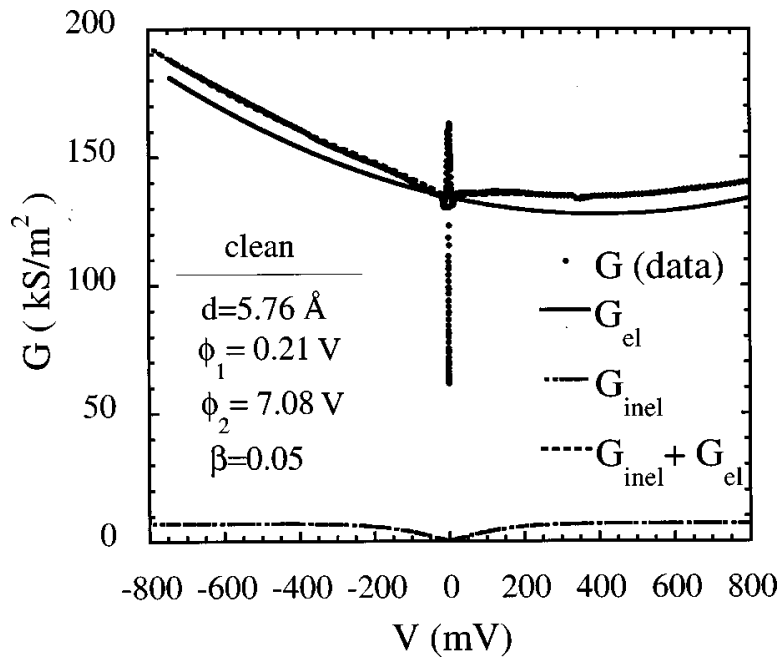

FIG. 5. The dynamic conductance fit for a clean $\mathrm{Al}-\mathrm{Al}_{2} \mathrm{O}_{3}-\mathrm{Pb}$ tunnel junction using elastic and inelastic tunneling components.

tic tunneling was found to be $170 \mathrm{mV}$ for all of the junctions analyzed. The barrier heights $\phi_{1}, \phi_{2}$, expressed in volts, the barrier thickness $d$ in angstroms, and the bias voltage value for the minimum conductance $V_{\text {min }}$ for each set of tunneling data were inferred from the fitting parameters $b, c$, and $\alpha$ by solving a nonlinear equation system consisting of

$$
\begin{aligned}
& \Delta \phi=\phi_{2}-\phi_{1}=\frac{9}{28} \frac{A_{0}^{2}}{c}, \\
& \bar{\phi}=\frac{\phi_{1}+\phi_{2}}{2}=\frac{16 \bar{\phi}^{3 / 2}}{A_{0} b}, \\
& G(0)=\left(3.16 \times \frac{4}{9} 10^{10} \frac{\sqrt{\bar{\phi}}}{d} \exp (-1.025 d \sqrt{\bar{\phi}})\right),
\end{aligned}
$$

and

$$
e V_{\min }=0.649 \frac{\Delta \phi}{d \sqrt{\bar{\phi}}},
$$

where $A_{0}=0.685 d$. The ratio of the maximum inelastic tunneling conductance to the minimum elastic tunneling conductance can be defined as

$$
\beta=\frac{G_{\text {inel }}\left(V_{\text {max }}\right)}{G_{\text {el }}\left(V_{\min }\right)}
$$

and this provides a quality factor to describe the junction. Ideal junctions would have $\beta=0$.

Using the procedure outlined above the elastic and inelastic tunneling contributions of the clean and modified $\mathrm{Al}-\mathrm{Al}_{2} \mathrm{O}_{3}-\mathrm{Pb}$ tunnel junctions have been obtained. Generally, the calculated total tunneling conductance of elastic and inelastic tunneling contributions give very good fits to the data to bias voltages $\pm 800 \mathrm{mV}$. Figure 5 shows the data analysis for a representative tunneling conductance vs bias voltage for the clean $\mathrm{Al}$ oxide barriers. In Fig. 5, the calculated elastic, inelastic and total tunnel contributions are presented as $G_{\text {el }}, G_{\text {inel }}$ and $G_{\text {inel }}+G_{\text {el }}$, respectively. The barrier thickness was found to be $5.76 \AA$ for the clean junction. The 


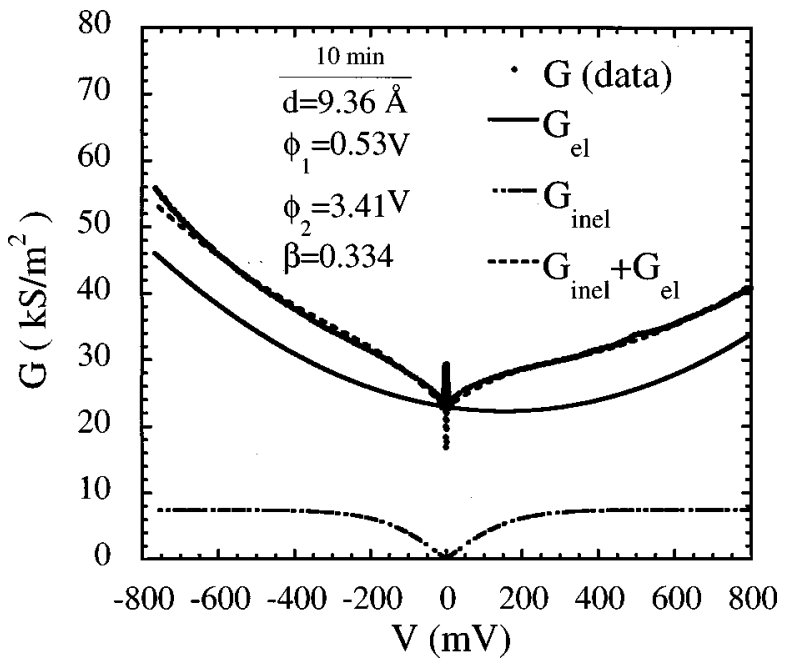

FIG. 6. The dynamic conductance fit for the modified $\mathrm{Al}-\mathrm{Al}_{2} \mathrm{O}_{3}-\mathrm{SAMs}-\mathrm{Pb}$ tunnel junction exposed to SAMs for $10 \mathrm{~min}$ using elastic and inelastic tunneling components.

barrier heights obtained for $\mathrm{Al}$ and $\mathrm{Pb}$ were 0.21 and $7.08 \mathrm{~V}$, respectively. The average barrier height was calculated as $3.65 \mathrm{~V}$. The conductance minimum was shifted from 0 to $406 \mathrm{mV}$ due to asymmetric barrier effects. There is a small inelastic tunneling conductance found due to possible hydrocarbon contaminations coming from the oil diffusion pump during the junction fabrication, but the ratio of inelastic tunneling to elastic tunneling was found to be $\beta=0.055$, which is quite low.

Figure 6 shows the data analysis for a representative tunneling conductance versus bias voltage for the modified Al-oxide barrier exposed to the OTMS molecules for 10 min. Using the same method as described before, the barrier thickness was found to be $9.36 \AA$ for the 10 min sample. The barrier heights for $\mathrm{Al}$ and $\mathrm{Pb}$ were obtained as 0.53 and 3.41 $\mathrm{V}$, respectively. The average barrier height was calculated as $1.98 \mathrm{~V}$. The minimum point was shifted from 0 to $155 \mathrm{mV}$

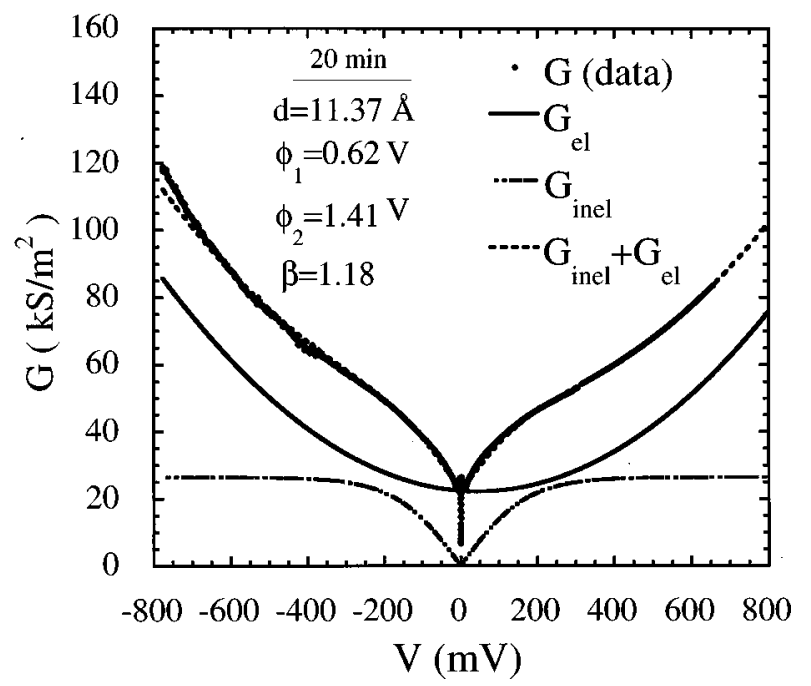

FIG. 7. The dynamic conductance fit for the modified $\mathrm{Al}-\mathrm{Al}_{2} \mathrm{O}_{3}-\mathrm{SAMs}-\mathrm{Pb}$ tunnel junction exposed to SAMs for $20 \mathrm{~min}$ using elastic and inelastic tunneling components.

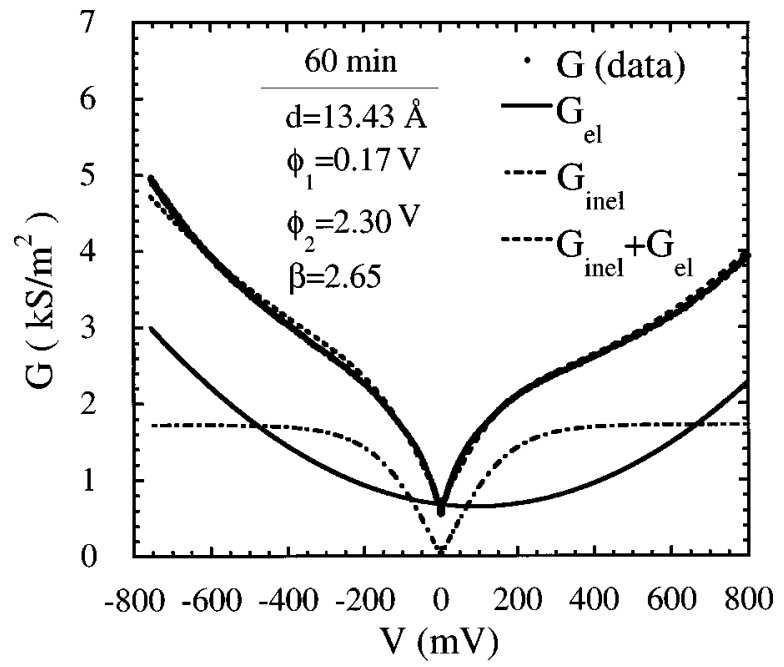

FIG. 8. The dynamic conductance fit for the modified $\mathrm{Al}-\mathrm{Al}_{2} \mathrm{O}_{3}-\mathrm{SAMs}-\mathrm{Pb}$ tunnel junction exposed to SAMs for $60 \mathrm{~min}$ using elastic and inelastic tunneling components.

due to asymmetric barrier effects. The inelastic tunneling conductance contribution started to increase due to introduced SAMs in the Al-oxide barrier. The inelastic tunneling ratio was found as $\beta=0.334$, which is six times that of the clean $\mathrm{Al}$-oxide tunnel barrier in Fig. 5. The saturation effect at $170 \mathrm{mV}$ of inelastic tunneling is more pronounced in the 10 min exposed $\mathrm{Al}$-oxide tunnel barrier conductance.

Figure 7 shows the data analysis for the tunneling conductance versus voltage for modified Al-oxide barriers exposed to SAMs for $20 \mathrm{~min}$. The barrier thickness was found to be $11.37 \AA$ for the $20 \mathrm{~min}$ sample. The barrier heights for $\mathrm{Al}$ and $\mathrm{Pb}$ were 0.62 and $1.14 \mathrm{~V}$, respectively. The average barrier height was calculated as $1.04 \mathrm{~V}$. The conductance minimum was found at $120 \mathrm{mV}$. The inelastic tunneling ratio increased to $\beta=1.18$.

The data analysis for the tunneling conductance versus bias voltage for the modified $\mathrm{Al}$-oxide barrier exposed to the OTMS molecules for 60 min is given in Fig. 8. The barrier

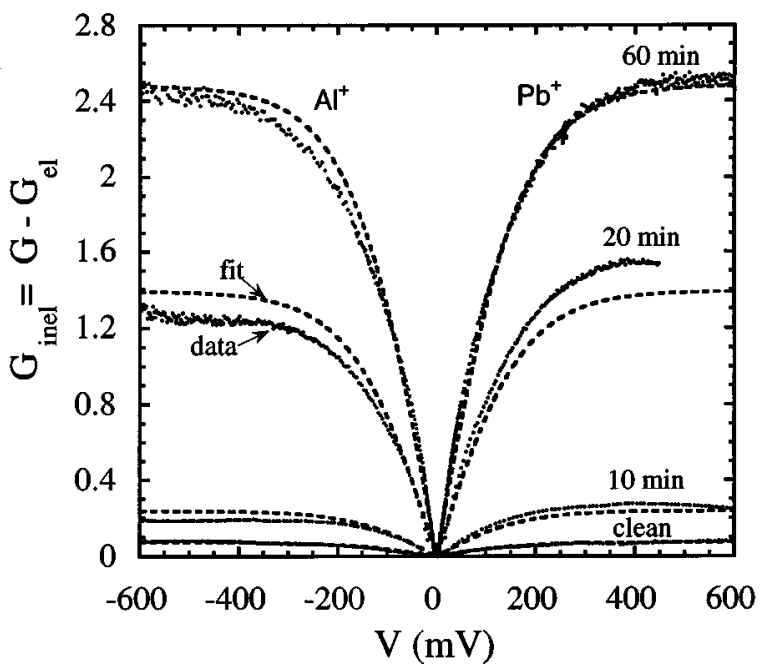

FIG. 9. The inferred inelastic conductance data comparison for clean and modified $\mathrm{Al}-\mathrm{Al}_{2} \mathrm{O}_{3}-\mathrm{SAMs}-\mathrm{Pb}$ tunnel junctions. Dashed lines are fits. 
TABLE I. $d I / d V$ analysis of clean and modified $\mathrm{Al}_{2} \mathrm{O}_{3}$ tunnel barriers.

\begin{tabular}{|c|c|c|c|c|c|c|c|}
\hline & Sample & $d(\AA)$ & $\phi_{1}(\mathrm{~V})$ & $\phi_{2}(\mathrm{~V})$ & $\bar{\phi}(\mathrm{V})$ & $G_{0}\left(\mathrm{kS} / \mathrm{m}^{2}\right)$ & $\beta$ \\
\hline \multirow{7}{*}{ clean } & $0 \mathrm{~A}$ & 7.77 & 0.033 & 4.03 & 1.99 & 74.0 & 0.087 \\
\hline & $0 \mathrm{~B}$ & 7.09 & 0.48 & 2.54 & 1.50 & 71.0 & 0.098 \\
\hline & $0 \mathrm{C}$ & 7.10 & 0.43 & 2.7 & 1.60 & 57.0 & 0.129 \\
\hline & OD & 7.14 & 0.34 & 2.83 & 1.59 & 56.0 & 0.120 \\
\hline & $0 \mathrm{E}$ & 7.07 & 0.22 & 2.72 & 1.47 & 82.0 & 0.120 \\
\hline & $0 \mathrm{~F}$ & 7.25 & 0.32 & 2.45 & 1.39 & 80.0 & 0.110 \\
\hline & $0 \mathrm{G}$ & 5.72 & 0.17 & 7.25 & 3.71 & 132 & 0.070 \\
\hline \multirow{5}{*}{$10 \mathrm{~min}$} & $10 \mathrm{~A}$ & 9.17 & 0.15 & 3.18 & 1.66 & 24.0 & 0.230 \\
\hline & $10 \mathrm{~B}$ & 9.66 & 0.30 & 2.15 & 1.20 & 63.0 & 0.200 \\
\hline & $10 \mathrm{C}$ & 8.58 & 0.39 & 3.23 & 1.81 & 36.0 & 0.238 \\
\hline & $10 \mathrm{D}$ & 9.36 & 0.53 & 3.41 & 1.98 & 23.0 & 0.334 \\
\hline & $10 \mathrm{E}$ & 8.89 & 0.31 & 3.20 & 1.75 & 23.0 & 0.302 \\
\hline \multirow{6}{*}{$20 \mathrm{~min}$} & $20 \mathrm{~A}$ & 11.47 & 0.25 & 1.75 & 1.06 & 20.0 & 1.370 \\
\hline & $20 \mathrm{~B}$ & 11.79 & 0.53 & 1.32 & 0.92 & 23.0 & 1.220 \\
\hline & $20 \mathrm{C}$ & 13.20 & 0.35 & 1.01 & 0.69 & 28.0 & 1.570 \\
\hline & 20D & 10.90 & 0.20 & 2.02 & 1.11 & 21.0 & 1.400 \\
\hline & $20 \mathrm{E}$ & 11.37 & 0.62 & 1.41 & 1.04 & 22.0 & 1.180 \\
\hline & $20 \mathrm{~F}$ & 13.73 & 0.34 & 0.94 & 0.64 & 23.0 & 1.980 \\
\hline \multirow{7}{*}{$60 \mathrm{~min}$} & $60 \mathrm{~A}$ & 11.07 & 0.83 & 2.57 & 1.68 & 0.404 & 2.22 \\
\hline & $60 \mathrm{~B}$ & 12.84 & 0.58 & 2.25 & 1.42 & 0.463 & 1.30 \\
\hline & $60 \mathrm{C}$ & 13.32 & 0.28 & 2.33 & 1.30 & 0.447 & 2.36 \\
\hline & 60D & 12.13 & 0.45 & 2.82 & 1.63 & 0.418 & 1.50 \\
\hline & $60 \mathrm{E}$ & 13.10 & 0.24 & 2.14 & 1.19 & 1.142 & 2.71 \\
\hline & $60 \mathrm{~F}$ & 12.35 & 0.29 & 2.30 & 1.30 & 1.595 & 2.49 \\
\hline & $60 \mathrm{G}$ & 13.43 & 0.17 & 2.30 & 1.20 & 0.712 & 2.65 \\
\hline
\end{tabular}

thickness increased to $13.43 \AA$ for the 60 min sample. The barrier heights for $\mathrm{Al}$ and $\mathrm{Pb}$ were obtained as 0.17 and 2.30 $\mathrm{V}$, respectively. The average barrier height was calculated as $1.2 \mathrm{~V}$. The minimum conductance was found at $95 \mathrm{mV}$ showing a lower asymmetry. The inelastic tunneling ratio increased to $\beta=2.65$. Again, the saturation effect at $170 \mathrm{mV}$ of inelastic tunneling is well pronounced. The superconducting $\mathrm{Pb}$ feature is barely visible.

The normalized inelastic tunneling conductances for the clean and modified Al-oxide barriers shown in Fig. 9 were obtained by subtracting the calculated elastic tunneling conductance term given in Eq. (1) from the original conductance data. The inferred inelastic tunneling conductance shows asymmetry with bias polarity. The inferred inelastic tunneling conductance with $\mathrm{Pb}$ positive bias is greater than that with $\mathrm{Al}$ positive bias in the modified $\mathrm{Al}$-oxide tunnel junctions. Our simple $\tanh \left(|V| / V_{c}\right)$ approximation for inelastic tunneling conductance explains the linear increasing background and saturation at $170 \mathrm{mV}$, but it fails in explaining the asymmetry with reverse polarity, $\operatorname{since} \tanh \left(|V| / V_{c}\right)$ is a symmetric function. Similar asymmetries were reported in several other inelastic tunneling experiments. ${ }^{16}$ In these experiments, however, the inelastic tunneling conductance was calculated finding the area under the peaks in $d^{2} I / d V^{2}$ corresponding the excitations in the barrier region. The peak intensities due to phonon excitation with the $\mathrm{Pb}$ electrode positive are always much greater than with the $\mathrm{Al}$ counterelectrode positive. ${ }^{16}$ The $( \pm)$ bias asymmetry in the inelastic tunneling intensity arises when the molecules are placed closer to one electrode than to the other since for one polarity the electron loses energy after tunneling and for the other polarity it loses energy before tunneling. The tunneling probability is proportional to energy. Thus as a consequence of spatial location of the interaction potential, the transmission probability for inelastic tunneling electron becomes asymmetric when the tunneling direction is reversed and a greater inelastic tunneling current and conductance with the $\mathrm{Pb}$ electrode positive are expected.

What is also evident in Fig. 9 is that the characteristic saturation voltage $V_{c}$ is essentially the same for all junctions, including those without any SAMs layer. This argues against any exotic explanations of the linear conductance channel such as the Coulomb correlation gap or those proposed for high $T_{c}$ superconductor junctions. ${ }^{17}$ Instead, the data suggest that within the framework of continuum inelastic tunneling, the excitations responsible for this channel exist in the barrier itself. One possibility is that the excitations are phonons associated with the $\mathrm{Al}$ oxide which, in the case of $\mathrm{Al}_{2} \mathrm{O}_{3}$, exist out to $120 \mathrm{meV}$. It is not unreasonable to imagine that for a nearly amorphous $\mathrm{Al}$-oxide barrier, ${ }^{18,19}$ these phonon modes would broaden out to higher energies and would exhibit a flat density of modes that would result in the linear increase in conductance observed. In this picture, the SAMs molecule simply acts as an activation center for the inelastic tunneling channel and the more molecules present on the oxide the greater the contribution from inelastic tunneling. Organic molecules typically have large dipole moments, which according to inelastic tunneling theory, increase the probability of an inelastic tunneling channel. ${ }^{20}$ This picture is also reasonable one to explain the small but non-negligible inelastic channel in the clean junctions. Here, organic molecules from contaminants in the vacuum system (roughing and diffusion pump oils) would act in the same way as the OTMS molecule.

The results from the junction analysis are shown in Table I. In general, there is an increase in the barrier thickness $d$ and parameter $\beta$ with increasing exposure time. The average barrier thickness increases from about $6 \AA$ for clean junctions to about $13 \AA$ for the 60 min exposure. The plots of $d$ and $\beta$ versus exposure are shown in Fig. 10 and they indicate that saturation of these parameter values has taken place

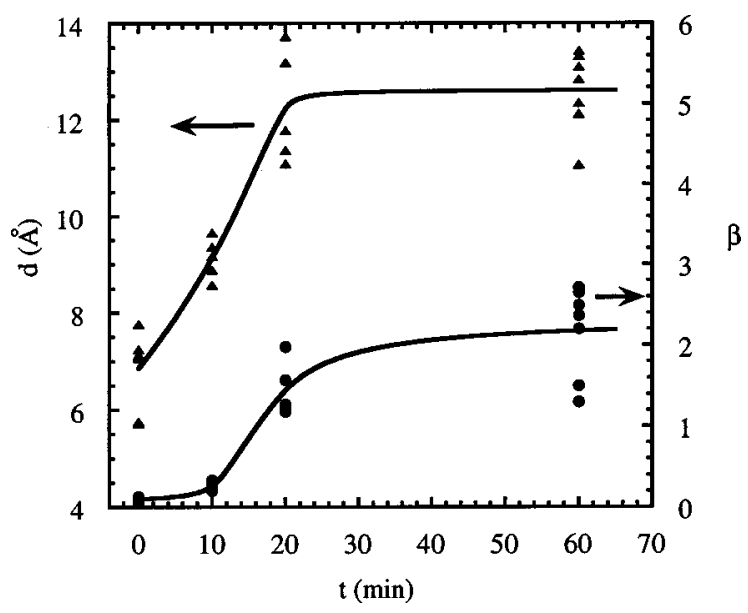

FIG. 10. The average barrier thickness vs exposure time (left hand side). The inelastic tunneling conductance ratio $\beta$ vs exposure time (right hand side). The lines are quides to the eye obtained by an interpolation routine. 
as would be expected for a nearly complete monolayer of the OTMS. The increase in barrier thickness is only $5 \AA$ with the SAMs layer which is small when compared to the OTMS length, but as mentioned in the literature ${ }^{21}$ the BDR theory tends to underestimate the actual barrier thickness by a factor of 2 .

The large value of $\beta$ for the 60 min exposure junctions indicates that inelastic tunneling is the dominant channel. This suggests that SAMs layers are not suitable candidates as elastic tunnel barriers for fundamental junction studies of superconductors or for device applications. However, the similarity of the linear conductance channel to that found on high $T_{c}$ superconductors as well as on other perovskite oxides ${ }^{17}$ suggests that ordinary barrier effects may be playing a role in those systems as well. Inelastic tunneling from a continuum of states may be a more common phenomenon than initially thought.

\section{ACKNOWLEDGMENTS}

The authors would like to acknowledge discussions with Liam Coffey and Jurgen Halbritter. S.O. would like to acknowledge Chad Mirkin, Dean Walter and Feng Xu, from the Organic Chemistry Department of Northwestern University for their valuable help in providing the organic molecule, octadecyltrimethoxysilane (OTMS) Self-Assembled Monolayers, and for their helpful suggestions. S.O. acknowledges Dr. Piner for providing AFM images of clean and modified Al-oxide surfaces. This work was partially supported by the National Science Foundation, Office of Science and Technology Centers under Contract No. DMR 91-20000.
${ }^{1}$ C. A. Mirkin, J. R. Valentine, D. Ofer, J. J. Hickman, and M. S. Wrighton, ACS Symp. Ser. 487, (1992).

${ }^{2}$ E. W. Wollman, D. Kang, C. D. Frisbie, I. M. Lorkovic, and M. S. Wrighton, J. Am. Chem. Soc. 116, 116 (1994).

${ }^{3}$ L. A. Bumm, J. J. Cygan, M. T. Dunver, and T. D. Burgin, Science 271, 1705 (1996)

${ }^{4}$ A. Ulman, Chem. Rev. 96, 1533 (1996).

${ }^{5}$ C. A. Mirkin, F. Xu, and J. Zhu, Adv. Mater. 9, 167 (1996).

${ }^{6}$ M. Covington, M. Aprili, E. Paraoanu, L. H. Greene, F. Xu, J. Zhu, and C. A. Mirkin, Phys. Rev. Lett. 79, 277 (1997).

${ }^{7}$ M. Covington, F. Xu, C. A. Mirkin, W. L. Feldmann, and L. H. Greene, Proceedings of the XXI International Conference on Low-temperature Physics, 1996.

${ }^{8}$ W. F. Brinkman, R. C. Dynes, and J. M. Rowell, J. Appl. Phys. 41, 1915 (1970).

${ }^{9}$ R. C. Jaklevic and J. Lambe, Phys. Rev. Lett. 17, 1139 (1966).

${ }^{10}$ J. Lambe and R. Jaklevic, Phys. Rev. 165, 821 (1968).

${ }^{11}$ P. K. Hansma and R. V. Coleman, Science 184, 1369 (1974).

${ }^{12}$ M. G. Simonsen, R. V. Coleman, and P. K. Hansma, J. Chem. Phys. 61, 3789 (1974).

${ }^{13}$ S. Okur, Ph.D. thesis, Illinois Institute of Technology, Chicago, 1998.

${ }^{14}$ J. R. Kirtley, S. Washburn, and D. J. Scalapino, Phys. Rev. B 45, 336 (1992).

${ }^{15}$ J. R. Kirtley and D. J. Scalapino, Phys. Rev. Lett. 65, 798 (1990).

${ }^{16}$ M. F. Muldoon, R. A. Dragoset, and R. V. Coleman, Phys. Rev. B 20, 416 (1979).

${ }^{17}$ H. Srikanth, K. P. Rajeev, G. V. Shivashankar, and A. K. Raychaudhuri, Physica C 195, 87 (1992).

${ }^{18}$ A. I. Braginski, J. Talvacchio, M. A. Janocko, and J. R. Gavaler, J. Appl. Phys. 60, 2058 (1986).

${ }^{19}$ F. King, in Aluminum and its Alloys, edited by E. G. West (Wiley, New York, 1987), Chap. 1.

${ }^{20}$ D. J. Scalapino and S. M. Marcus, Phys. Rev. Lett. 18, 459 (1967).

${ }^{21}$ E. L. Wolf, Principles of Electron Tunneling Spectroscopy (Oxford University Press, Oxford, 1985). 